\title{
A reliable algorithm for time-fractional Navier-Stokes equations via Laplace transform
}

https://doi.org/10.1515/nleng-2018-0080

Received May 23, 2018; revised August 20, 2018; accepted November 6, 2018.

\begin{abstract}
In this paper, numerical solution of fractional order Navier-Stokes equations in unsteady viscous fluid flow is found using q-homotopy analysis transform scheme. Fractional derivative is considered in Caputo sense. The proposed technique is a blend of q-homotopy analysis scheme and transform of Laplace. It executes well in efficiency and provides $h$-curves that show convergence range of series solution.
\end{abstract}

Keywords: Navier-Stokes (N-S) equations; q-homotopy analysis transform method (q-HATM); Caputo fractional derivative

\section{Introduction}

Every phenomenon in fields of Science and Engineering may be alternately modelled using fractional order derivatives. It is due to their non-local property, intrinsic to several complex systems. They are used as modelling tools in nanotechnology, viscoelasticity, anomalous transport, control theory, financial \& biological modelling etc. The most important among such models are those described by arbitrary order PDEs. Adomian decomposition [1], homotopy analysis, residual power series, fractional reduced differential transform, fractional variational iteration method [2-4], Laplace homotopy technique [5], Laplace variational iteration method [6], homotopy perturbation transform method [7], q-homotopy analysis transform method [8-10], modified trial equation method [11], new iterative Sumudu transform method [12] and Laplace perturbation method [13] etc. are some impor-

*Corresponding Author: Amit Prakash, Department of Mathematics, National Institute of Technology, Kurukshetra 136119, India, E-mail: amitmath@nitkkr.ac.in, amitmath0185@gmail.com Doddabhadrappla Gowda Prakasha, Pundikala Veeresha, Department of Mathematics, Karnatak University, Dharwad580003, India, E-mail: dgprakasha@kud.ac.in (D. G. Prakasha), viru0913@gmail.com (P. Veeresha) tant methods which are applied to find numerical solution of these problems.

$\mathrm{N}-\mathrm{S}$ equation define motion [14] of incompressible Newtonian fluid flow extending from enormous scale atmospheric motions to lubrication of bearings and express conservation of mass and momentum. Consider an unsteady, unidimensional viscous fluid motion in a long circular pipe. Fluid is initially at rest. Constant pressure gradient along pipe axis is abruptly imposed to set fluid in motion. Flow is taken as axially symmetric. It is given in [15] as,

$$
\rho u_{t}=-p_{z}+\mu\left(D_{r}^{2} u+\frac{1}{r} D_{r} u\right),
$$

axis of pipe is z-axis and $r$ is radial direction.

El-Shashed and Salem [16] generalized N-S Eq. by fractional N-S Eq. of order $\alpha$, as

$$
D_{t}^{\alpha} u=P+\mu\left(D_{r}^{2} u+\frac{1}{r} D_{r} u\right), 0<\alpha \leq 1,
$$

where $P=-\frac{\partial p}{\rho \partial z}, \alpha$ is order of fractional derivative. If $\alpha=1$, fractional N-S Eq. reduces to classical N-S equation. Here, efficiency of q-HATM is considered to numerically solve time-fractional N-S Eq. (2). It is an elegant union of q-HAM $[17,18]$ \& transform of Laplace. The q-HAM is actually improvement of parameter $q \in[0,1]$ in HAM to $q \in\left[0, \frac{1}{n}\right], n \geq 1$. Its authority is its potential of adjusting two strong computational methodologies for probing FDEs. Eq. (2) has been handled by Momani and Odibat by ADM [19], Khan et al. by HPM and variation iteration method [20], Ragab et al. by HAM [21], Kumar et al. by fractional modified Laplace decomposition method [22] and HPTM [23], Wang and Liu by modified reduced differential transform method [24] etc. But it has not been solved by q-HATM.

\section{Preliminaries}

Definition 2.1. A real valued function $f(t), t>0$ is in space $C_{\mu}, \mu \in R$ if $\exists$ a real number $p(>\mu)$ s.t. $f(t)=t^{p} f_{1}(t) ; f_{1} \in$ $C[0, \infty)$, and is in space $C_{\mu}^{m}$ if $f^{m} \in C_{\mu}, M \in N \bigcup\{0\}[25$, 26]. 
Definition 2.2. Caputo fractional derivative [9] of $f, f \in$ $C_{-1}^{m}, m \in N \bigcup\{0\}$ is

$$
D_{t}^{\alpha} f(t)=\left\{\begin{array}{c}
\frac{d^{n} f(t)}{d t^{n}}, \quad \alpha=n \in N, \\
\frac{1}{\Gamma(n-\alpha)} \int_{0}^{t}(t-\tau)^{n-\alpha-1} f^{n}(\tau) d \tau, \\
n-1<\alpha<n, n \in N .
\end{array}\right.
$$

Definition 2.3. Laplace transform (LT) of Caputo fractional derivative is

$$
\begin{gathered}
L\left[D_{t}^{n \alpha} u(r, t)\right]=s^{n \alpha} L[u(r, t)]-\sum_{k=0}^{n-1} s^{n \alpha-k-1} u^{k}(r, 0), \\
n-1<n \alpha \leq n .
\end{gathered}
$$

\section{Implementation of q-HATM on Navier-Stokes equation}

To illustrate its efficiency, we take a fractional nonlinear nonhomogeneous PDE,

$$
D_{t}^{\alpha} u(r, t)+R u(r, t)+N u(r, t)=f(r, t), \quad n-1<\alpha \leq n,
$$

where $D_{t}^{\alpha} u$ is Caputo fractional derivative, $R, N$ are linear, nonlinear differential operators, $f(r, t)$ is source term.

Taking LT on Eq. (3) and applying its differentiation property, we get

$$
\begin{aligned}
& L[u(r, t)]-\frac{1}{s^{\alpha}} \sum_{k=0}^{n-1} s^{\alpha-k-1} u^{k}(r, 0) \\
& +\frac{1}{s^{\alpha}}\{L[R u(r, t)]+L[N u(r, t)]-L[f(r, t)]\}=0 .
\end{aligned}
$$

Nonlinear operator $\mathrm{N}$ is

$$
\begin{aligned}
& N[\varphi(r, t ; q)]=L[\varphi(r, t ; q)]-\frac{1}{s^{\alpha}} \sum_{k=0}^{n-1} s^{\alpha-k-1} \varphi^{k}(r, t ; q)\left(0^{+}\right) \\
& +\frac{1}{s^{\alpha}}\{L[R \varphi(r, t ; q)]+L[N \varphi(r, t ; q)]-L[f(r, t)]\}, \quad \text { (5) }
\end{aligned}
$$

here $q \in\left[0, \frac{1}{n}\right]$ and $\phi$ is real function.

Build homotopy as

$$
\begin{gathered}
(1-n q) L\left[\varphi(r, t ; q)-u_{0}(r, t)\right]=h q H(r, t) N[\varphi(r, t ; q)], \\
n \geq 1
\end{gathered}
$$

$q$ is embedding parameter, $H \neq 0$ is auxiliary function, $h \neq 0$ is auxiliary parameter, $u_{0}$ is initial guess.

For $=0, \frac{1}{n}$; following results holds:

$$
\varphi(r, t ; 0)=u_{0}(r, t), \varphi\left(r, t ; \frac{1}{n}\right)=u(r, t) .
$$

As $q$ increases, $\varphi$ varies from $u_{0}$ to solution $u(r, t)$. Expanding $\varphi$ about $q$ by Taylor's theorem, we get

$$
\varphi(r, t ; q)=u_{0}+\sum_{m=1}^{\infty} u_{m}(r, t) q^{m},
$$

where

$$
u_{k}(r, t)=\left.\frac{1}{k !} \frac{\partial^{k} \varphi(r, t ; q)}{\partial q^{k}}\right|_{q=0} .
$$

With suitable choice of $u_{0}, n, \hbar, H$, series (8) converges at $q=\frac{1}{n}$, we get

$$
u(r, t)=u_{0}+\sum_{m=1}^{\infty} u_{m}(r, t)\left(\frac{1}{n}\right)^{m}
$$

Define vectors as

$$
\vec{u}_{m}=\left\{u_{0}(r, t), u_{1}(r, t), \ldots, u_{m}(r, t)\right\} .
$$

Differentiating deformation Eq. (6) $m$-times w. r. t $q$, dividing by $m$ ! \& taking $q=0$, we get

$$
L\left[u_{m}(r, t)-k_{m} u_{m-1}(r, t)\right]=\hbar H(r, t) R_{m}\left(\vec{u}_{m-1}\right) .
$$

Using inverse transform, we get

$$
u_{m}(r, t)=k_{m} u_{m-1}(r, t)+\hbar L^{-1}\left[H(r, t) R_{m}\left(\vec{u}_{m-1}\right)\right],
$$

where

$$
R_{m}\left(\vec{u}_{m-1}\right)=\left.\frac{1}{(m-1) !} \frac{\partial^{m-1} N[\varphi(r, t ; q)]}{\partial q^{m-1}}\right|_{q=0},
$$

and

$$
k_{s}=\left\{\begin{array}{l}
0, s \leq 1 \\
n, s>1
\end{array}\right.
$$

\section{Numerical Experiments}

Now we implement this method on few test problems.

Example 4.1. Consider a time-fractional N-S equation

$$
D_{t}^{\alpha} u=D_{r}^{2} u+\frac{1}{r} D_{r} u, \quad 0<\alpha \leq 1,
$$

with initial condition

$$
u(r, 0)=r .
$$

Taking transform of Laplace on each side of Eq. (16) and simplifying, we get

$$
L[u]-\frac{r}{s}-\frac{1}{s^{\alpha}} L\left[D_{r}^{2} u+\frac{1}{r} D_{r} u\right]=0 .
$$


$\mathrm{N}$ is defined as,

$$
\begin{aligned}
& N[\varphi(r, t ; q)]=L[\varphi(r, t ; q)]-\left(1-\frac{k_{m}}{n}\right) \frac{r}{s} \\
& -\frac{1}{s^{\alpha}} L\left[\frac{\partial^{2} \varphi(r, t ; q)}{\partial r^{2}}+\frac{1}{r} \frac{\partial \varphi(r, t ; q)}{\partial r}\right] .
\end{aligned}
$$

The $m^{\text {th }}$-order deformation eqn. for $H=1$ is

$$
L\left[u_{m}(r, t)-k_{m} u_{m-1}(r, t)\right]=\hbar R_{m}\left(\vec{u}_{m-1}\right),
$$

where

$$
\begin{aligned}
& R_{m}\left(\vec{u}_{m-1}\right)=L\left[u_{m-1}\right]-\left(1-\frac{k_{m}}{n}\right) \frac{r}{s} \\
& -\frac{1}{s^{\alpha}} L\left[\frac{\partial^{2} u_{m-1}}{\partial r^{2}}+\frac{1}{r} \frac{\partial u_{m-1}}{\partial r}\right] .
\end{aligned}
$$

Taking inverse transform on Eq. (20), we find

$$
u_{m}=k_{m} u_{m-1}+\hbar L^{-1}\left\{R_{m}\left(\vec{u}_{m-1}\right)\right\} .
$$

Taking $u_{0}$ and solving, we get

$$
\begin{gathered}
u_{1}=\frac{-\hbar t^{\alpha}}{r \Gamma(\alpha+1)}, \\
u_{2}=\frac{-(n+\hbar) \hbar t^{\alpha}}{r \Gamma(\alpha+1)}+\frac{\hbar^{2} t^{2 \alpha}}{r^{3} \Gamma(\alpha+1)}, \\
u_{3}=\frac{-(n+\hbar)^{2} \hbar t^{\alpha}}{r \Gamma(\alpha+1)}+\frac{2(n+\hbar) \hbar^{2} t^{2 \alpha}}{r^{3} \Gamma(2 n+1)}-\frac{9 \hbar^{3} t^{3 \alpha}}{r^{5} \Gamma(3 \alpha+1)}, \ldots
\end{gathered}
$$

Hence, next values may be got. The series solution of Eq. (16) is

$$
u(r, t)=u_{0}+\sum_{m=1}^{\infty} u_{m}(r, t)\left(\frac{1}{n}\right)^{m} .
$$

If $n=1, \hbar=-1$ in Eq. (23), we arrive at

$$
u(r, t)=r+\sum_{n=1}^{\infty} \frac{1^{2} \cdot 3^{2} \ldots(2 n-3)^{2}}{r^{2 n-1}} \frac{t^{n \alpha}}{n !} .
$$

For $\alpha=1$ in Eq. (24), we gain solution of standard N-S equation as

$$
u(r, t)=r+\sum_{n=1}^{\infty} \frac{1^{2} \cdot 3^{2} \ldots(2 n-3)^{2}}{r^{2 n-1}} \frac{t^{n}}{n !},
$$

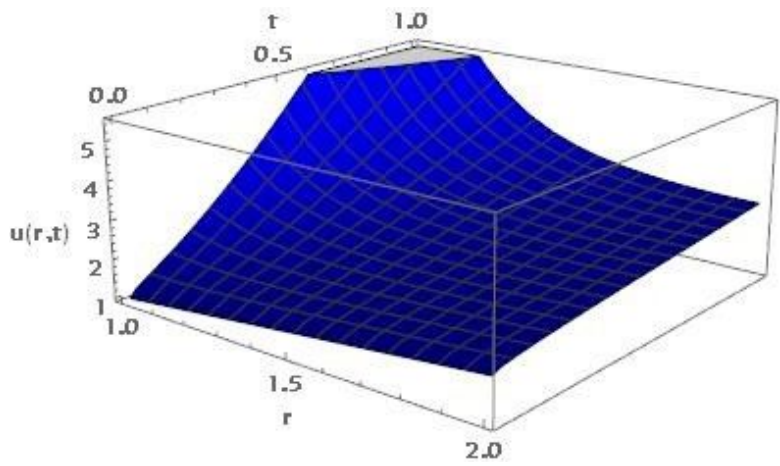

(a)

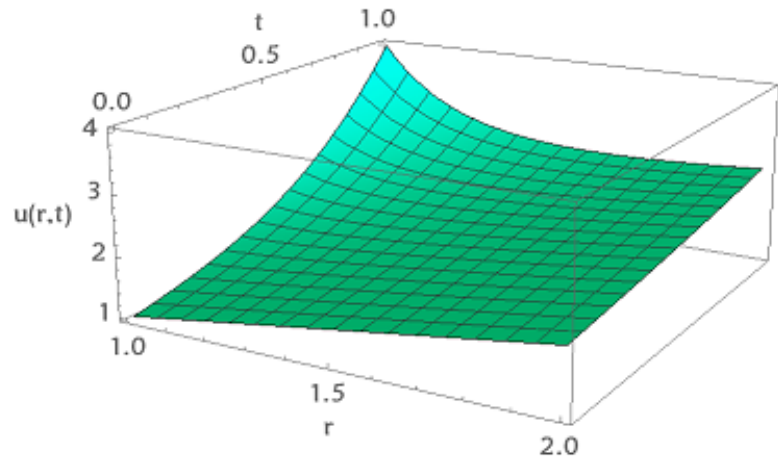

(b)

Fig. 1: Plots of q-HATM solution $u(r, t)$ Vs. $r, t$ when $\hbar=-1, n=1$, for (a) $\alpha=0.5$ (b) $\alpha=1$, for Ex. 4.1

Example 4.2. Now, consider time-fractional N-S equation

$$
D_{t}^{\alpha} u=P+D_{r}^{2} u+\frac{1}{r} D_{r} u, 0<\alpha \leq 1,
$$

with initial condition

$$
u(r, 0)=1-r^{2} .
$$

Taking LT on Eq. (25) and simplifying, we get

$$
L[u]-\frac{\left(1-r^{2}\right)}{s}-\frac{P}{s^{\alpha+1}}-\frac{1}{s^{\alpha}} L\left[D_{r}^{2} u+\frac{1}{r} D_{r} u\right]=0 .
$$

Also,

$$
\begin{aligned}
& N[\varphi(r, t ; q)]=L[\varphi(r, t ; q)]-\left(1-\frac{k_{m}}{n}\right) \frac{\left(1-r^{2}\right)}{s} \\
& -\frac{P}{s^{\alpha+1}}-\frac{1}{s^{\alpha}} L\left[\frac{\partial^{2} \varphi(r, t ; q)}{\partial r^{2}}+\frac{1}{r} \frac{\partial \varphi(r, t ; q)}{\partial r}\right] .
\end{aligned}
$$

For $H=1$, deformation equation is

$$
L\left[u_{m}(r, t)-k_{m} u_{m-1}(r, t)\right]=\hbar R_{m}\left(\vec{u}_{m-1}\right),
$$


Table 1: Comparison of results by HAM, FMLDM, HPTM, q-HATM at different values of $r$ and $t$ when $\alpha=1, \hbar=-1, n=1$ for Ex. 4.1.

\begin{tabular}{cccccc}
\hline $\boldsymbol{r}$ & $\boldsymbol{t}$ & HAM[21] & FMLDM [22] & HPTM [23] & q-HATM \\
\hline 1.25 & & 1.4736800000 & 1.4736800000 & 1.4736800000 & 1.4736800000 \\
1.50 & 0.25 & 1.6790123457 & 1.6790123457 & 1.6790123457 & 1.6790123457 \\
1.75 & & 1.9001160231 & 1.9001160231 & 1.9001160231 & 1.9001160231 \\
2.00 & & 2.1296386719 & 2.1296386719 & 2.1296386719 & 2.1296386719 \\
\hline 1.25 & & 1.7754400000 & 1.7754400000 & 1.7754400000 & 1.7754400000 \\
1.50 & 0.50 & 1.8950617284 & 1.8950617284 & 1.8950617284 & 1.8950617284 \\
1.75 & & 2.0704617124 & 2.0704617124 & 2.0704617124 & 2.0704617124 \\
2.00 & & 2.2714843750 & 2.2714843750 & 2.2714843750 & 2.2714843750 \\
\hline 1.25 & & 2.2013600000 & 2.2013600000 & 2.2013600000 & 2.2013600000 \\
1.50 & 0.75 & 2.1666666667 & 2.1666666667 & 2.1666666667 & 2.1666666667 \\
1.75 & & 2.2696049265 & 2.2696049265 & 2.2696049265 & 2.2696049265 \\
2.00 & & 2.4299316406 & 2.4299316406 & 2.4299316406 & 2.4299316406 \\
\hline 1.25 & & 2.7975200000 & 2.7975200000 & 2.7975200000 & 2.7975200000 \\
1.50 & 1 & 2.5123456790 & 2.5123456790 & 2.5123456790 & 2.5123456790 \\
1.75 & 1 & 2.5061135241 & 2.5061135241 & 2.5061135241 & 2.5061135241 \\
2.00 & & 2.6093750000 & 2.6093750000 & 2.6093750000 & 2.6093750000 \\
\hline
\end{tabular}

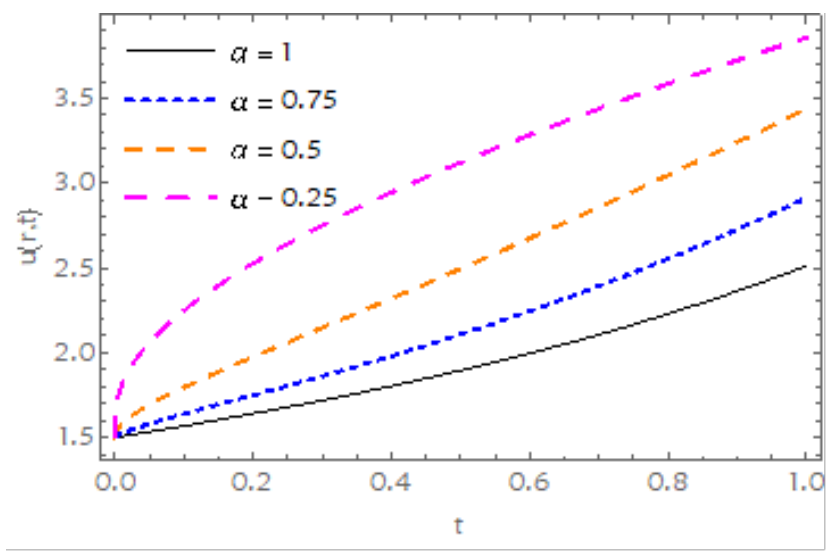

Fig. 2: $\alpha$-curve of solution $u(r, t)$ Vs. $t$ when $\hbar=-1, n=1, r=1.5$ for Ex. 4.1

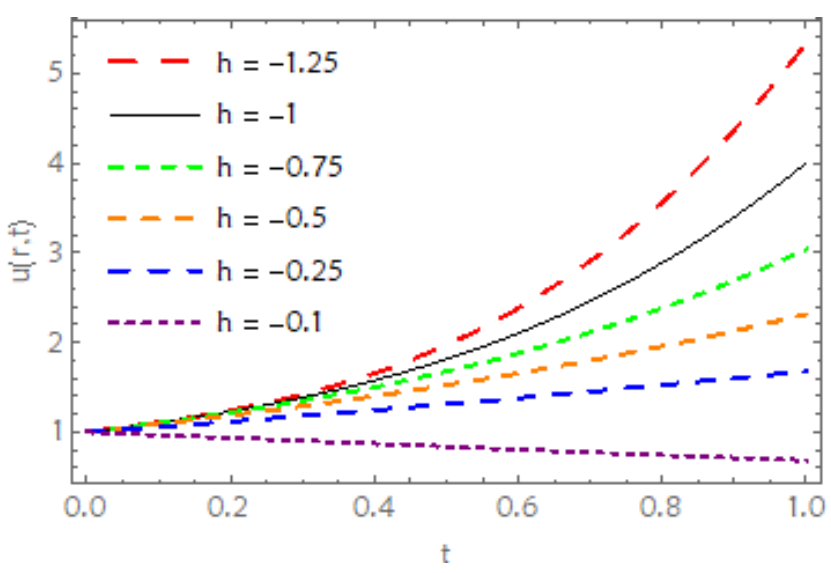

Fig. 3: Plot of solution $u(r, t)$ Vs. $t$ when $n=1=\alpha=r$ for Ex. 4.1

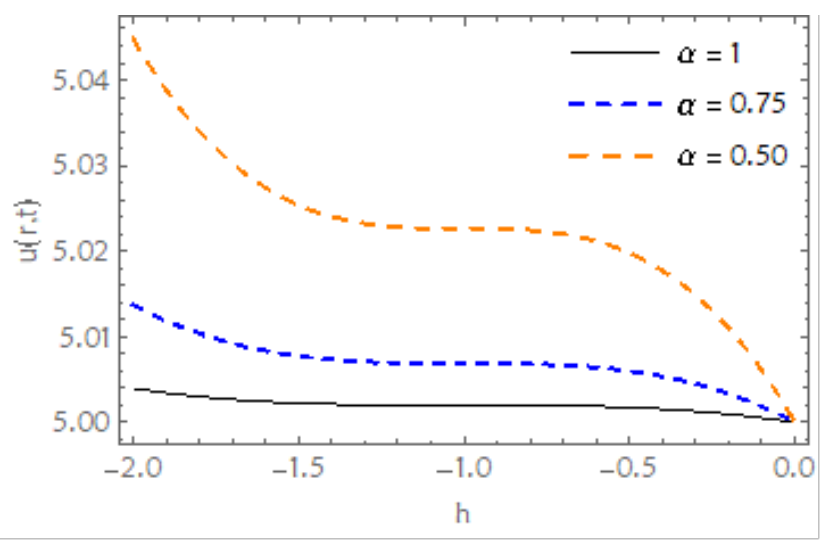

Fig. 4: $\hbar$-curve of q-HATM solution $u(r, t)$ Vs. $h$ when $t=0.01, r=$ $1, n=1$ for Ex. 4.1

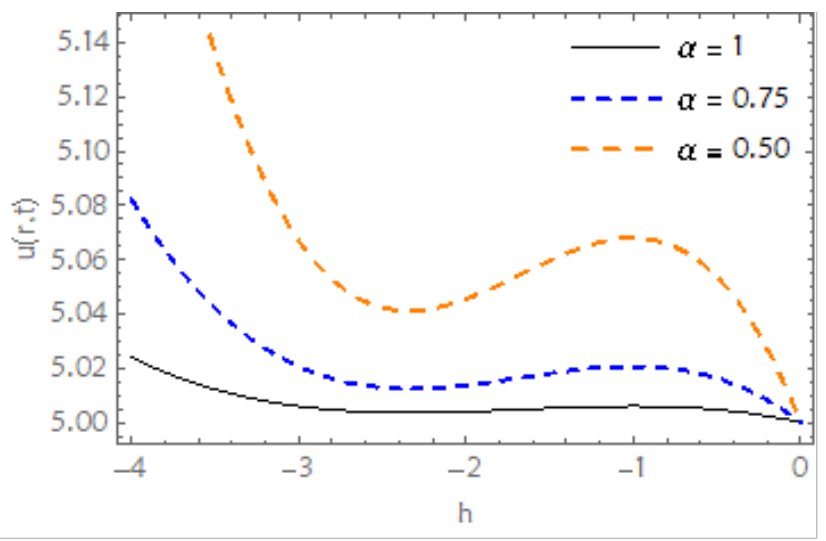

Fig. 5: $\hbar$-curve of q-HATM solution $u(r, t)$ Vs. $h$ when $t=0.01, r=$ $1, n=2$ for Ex. 4.1 


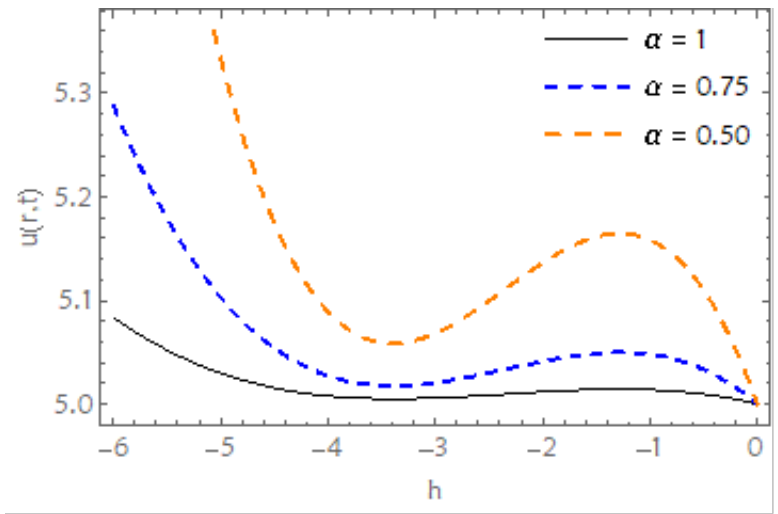

Fig. 6: $\hbar$-curve of q-HATM solution $u(r, t)$ Vs. $h$ when $t=0.01, r=$ 1, $n=3$ for Ex. 4.1

Here

$$
\begin{aligned}
& R_{m}\left(\vec{u}_{m-1}\right)=L\left[u_{m-1}\right]-\left(1-\frac{k_{m}}{n}\right) \frac{\left(1-r^{2}\right)}{s} \\
& -\frac{P}{s^{\alpha+1}}-\frac{1}{s^{\alpha}} L\left[\frac{\partial^{2} u_{m-1}}{\partial r^{2}}+\frac{1}{r} \frac{\partial u_{m-1}}{\partial r}\right] .
\end{aligned}
$$

By inverse transform,

$$
u_{m}=k_{m} u_{m-1}+\hbar L^{-1}\left\{R_{m}\left(\vec{u}_{m-1}\right)\right\}
$$

Taking $u_{0}$ and solving, we get

$$
\begin{gathered}
u_{1}=\frac{\hbar(4-P) t^{\alpha}}{\Gamma(\alpha+1)}, \\
u_{2}=\frac{[(n+\hbar)(4-P)-P] \hbar t^{\alpha}}{\Gamma(\alpha+1)}, \\
u_{3}=\frac{\left[(n+\hbar)^{2}(4-P)-(n+\hbar) P-P\right] \hbar t^{\alpha}}{\Gamma(\alpha+1)},
\end{gathered}
$$

and so on ....

Series solution of Eq. (25) is

$$
u(r, t)=u_{0}+\sum_{m=1}^{\infty} u_{m}(r, t)\left(\frac{1}{n}\right)^{m} .
$$

\section{Numerical simulations and discussion}

Figs. 1 and 7 show plots of numerical results of Eqs. (16) and (25) respectively when $\alpha=0.5$ and 1 for Ex. 4.1 and 4.2. Fig. 2 displays behaviour of solution for arbitrary order $\alpha$ and standard case $\alpha=1$ at $r=0.5, \hbar=-1, n=1$ for Ex. 4.1. Fig. 8 shows behaviour of solution for arbitrary order

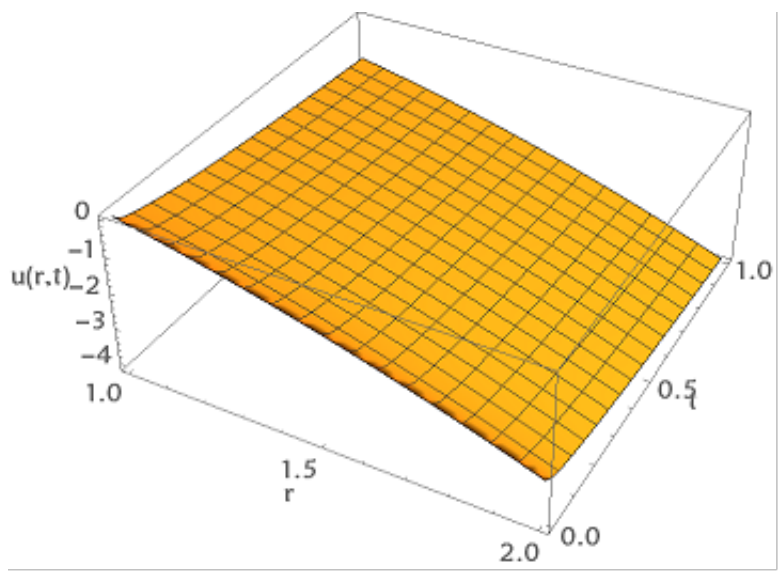

(a)

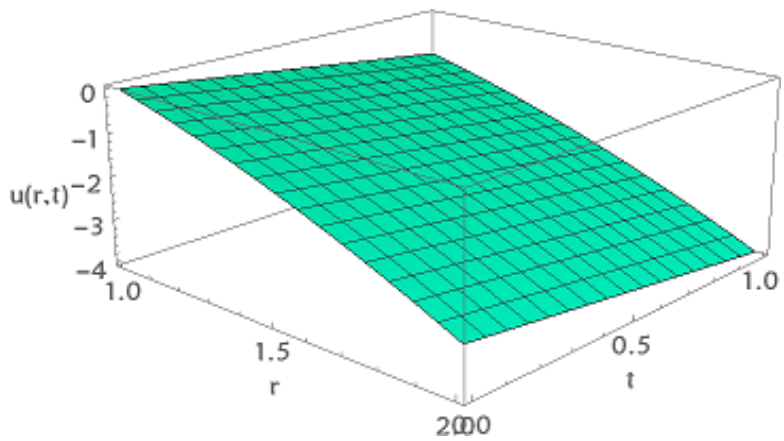

(b)

Fig. 7: Plots of q-HATM solution $u(r, t)$ Vs. $r, t$ when $P=1, \hbar=$ $-1, n=1$, (a) $\alpha=0.5$, (b) $\alpha=1$, for Ex. 4.2.

$\alpha$ and standard case $\alpha=1$ at $r=1.5, \hbar=-1, \quad n=1=P$ for Ex. 4.2. It is clear from Fig. 2 and 8 that as $\alpha$ tends to 1, the q-HATM solution converges. Fig. 3 and 9 represent convergence control parameter curves for Ex. 4.1 and 4.2. The value of $\hbar$ should be negative. From Fig. 3 and 9, it is clear that, as $\hbar$ decreases, rate of covergence increases. Figs. 4-6 and 10-12, show $\hbar$-curves at distinct order of fractional derivative at $n=1,2,3$ for Ex. 4.1 and 4.2. In h-curves, horizontal line exhibits convergence range of solution. We observe that as arbitrary order of derivative increases, range of convergence increases. Also, from Figs. 4-6, 10-12, it is clear that range of convergence depends positively on $\mathrm{n}$. Table 1 shows comparison of results by HAM, FMLDM, HPTM, q-HATM at different values of rand $t$ when $\alpha=1=n, \hbar=-1$ for Ex. 4.1. It can be observed from Table 1 that our results are accurate and agree with existing methods. 


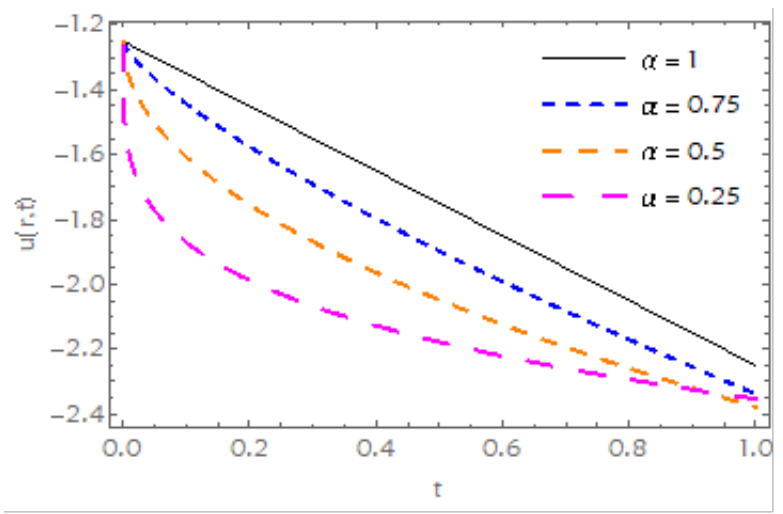

Fig. 8: $\alpha$-curve of solution $u(r, t)$ Vs. $t$ when $P=1, \hbar=-1, n=1, r=$ 1.5 for Ex. 4.2.

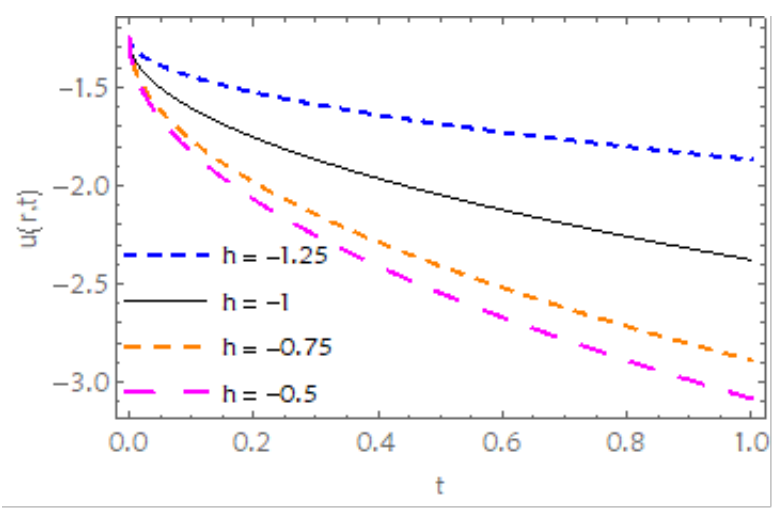

Fig. 9: Plot of solution $u(r, t)$ Vs. $t$ when $P=1, n=1, \alpha=1, r=1$, for Ex. 4.2.

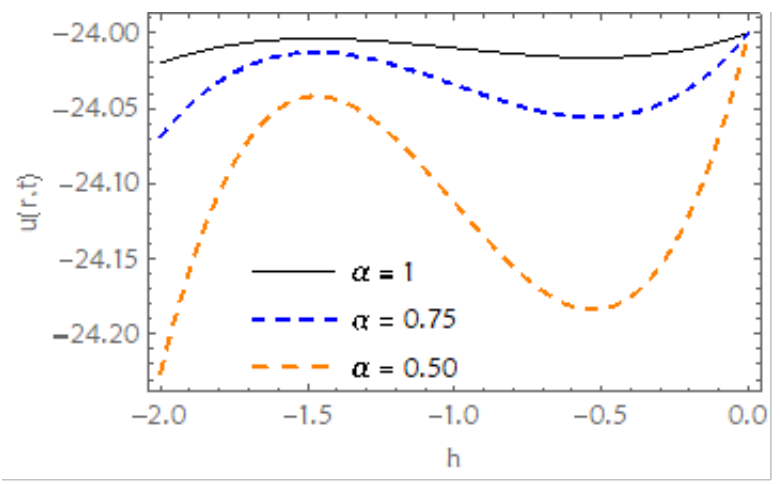

Fig. 10: $h$-curve of solution $u(r, t)$ Vs. $\hbar$ obtained when $t=$ $0.01, r=1, n=1$, for Ex. 4.2

\section{Conclusion}

In this paper, approximate analytic solution of timefractional N-S equation is gained by the q-HATM. It is worth mentioning that q-HATM is capable of reducing time and work of computation in comparison to existing numerical methods keeping higher accuracy of results intact. The

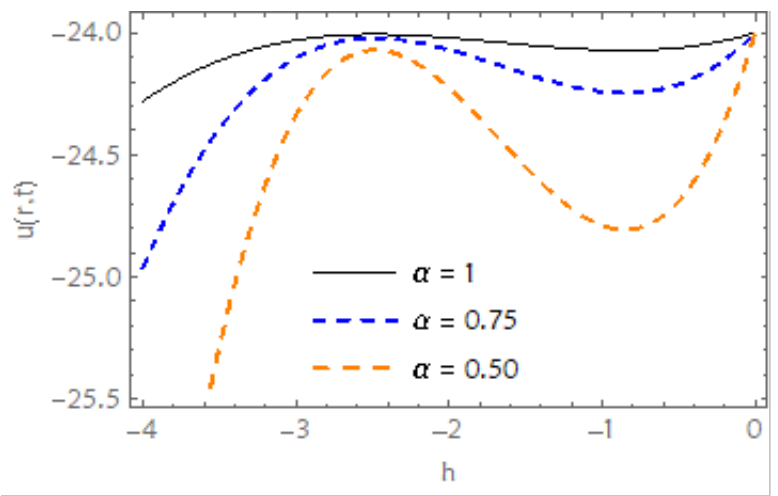

Fig. 11: $h$-curve of solution $u(r, t)$ Vs. $\hbar$ obtained when $t=0.01, r=$ $1, n=2$ for Ex. 4.2

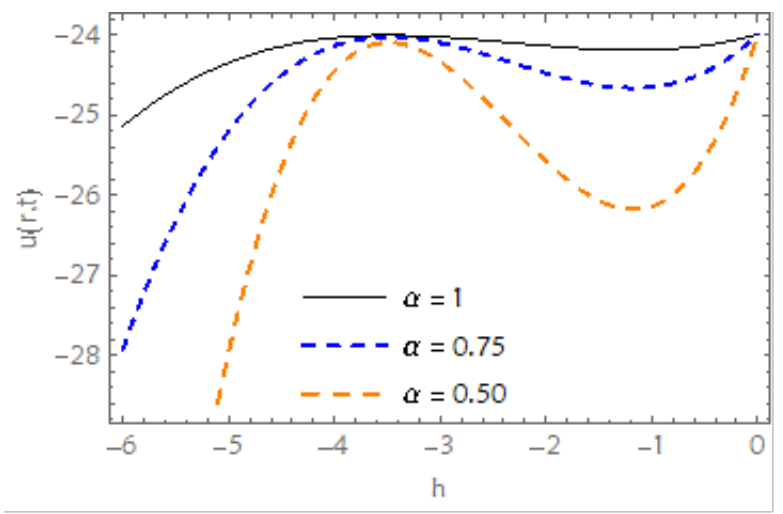

Fig. 12: $h$-curve of solution $u(r, t)$ Vs. $\hbar$ when $t=0.01, r=1, n=$ 3 for Ex. 4.2

q-HATM contains parameters $\hbar$ and $n$, that can be adopted to manage convergence of solution. It makes this scheme more powerful and exciting.

Acknowledgement: The authors are extremely thankful to the reviewer's for carefully reading the paper and useful comments and suggestions which have helped to improve the paper.

\section{References}

[1] S. Momani and R. Qaralleh, Numerical approximations and Pade approximants for a fractional population growth model, Appl. Math. Model., 31 (2007) 1907-1914.

[2] A. Prakash and M. Kumar, Numerical solution of two dimensional time-fractional order biological population model, Open Physics, 14 (2016) 177-186.

[3] A. Prakash, M. Goyal and S. Gupta, Fractional variation iteration method for solving time - fractional NewellWhitehead-Segel equation, Nonlinear Engineering, (2018) 1-10, https://doi.org/10.1515/nleng-2018-0001. 
[4] A. Prakash, M. Kumar and K. K. Sharma, Numerical method for solving coupled Burgers equation, Applied Mathematics and Computation, 260 (2015) 314-320.

[5] M. Yavuz and N. Ozdemir, Numerical inverse Laplace homotopy technique for fractional heat equations, Therm. Sci., 22(1) (2018) 185-194.

[6] Z. Hammouch and T. Mekkaoui, A Laplace-variational iteration method for solving the homogeneous Smoluchowski coagulation equation, Applied Mathematical Sciences, 6 (18) (2012) $879-886$.

[7] A. Prakash, Analytical method for space-fractional telegraph equation by Homotopy perturbation transform method, Nonlinear Engineering, 5(2) 2016 123-128.

[8] D. Kumar, J. Singh, D. Baleanu, A new analysis for fractional model of regularized long-wave equation arising in ion acoustic plasma waves, Math. Meth. Appl. Sci., 40 (2017) 56425653.

[9] A. Prakash and H. Kaur, Numerical solution for fractional model of Fokker-Plank equation by using q-HATM, Chaos, Solitons \& Fractals, 105 (2017) 99-110.

[10] A. Prakash and H. Kaur, q-Homotopy analysis transform method for space- and time-fractional nonlinear KdV-Burgers equations, Nonlinear Sci. Lett. A, 9(1) (2018) 44-61.

[11] H. Bulut, H. M. Baskonus and Y. Pandir, The modified trial equation method for fractional wave equation and time fractional generalized Burgers equation, Abstract and Applied Analysis, Vol. 2013 (2013) 1-8, http://dx.doi.org/10.1155/2013/ 636802.

[12] A. Prakash, M. Kumar and D. Baleanu, A new iterative technique for a fractional model of nonlinear Zakharov-Kuznetsov equations via Sumudu transform, Applied Mathematics and Computation, 334 (2018) 30-40.

[13] M. Yavuz, N. Ozdemir and H. M. Baskonus, Solutions of partial differential equations using the fractional operator involving Mittag-Leffler kernel, Eur. Phys. J. Plus, 133(6) (2018) 215.

[14] P. G. Lemarie Rieusset, Recent developments in the NavierStokes problem, CRC Press, USA, 2002.
[15] J. L.Bansal, Viscous Fluid Dynamics, Oxford, 1997.

[16] M. El-Shahed and A. Salem, On the generalized Navier-Stokes equations, Appl. Math. Comput., 156(1) (2004) 287-293.

[17] M. A. El-Tawil and S. N. Huseen, The q-Homotopy analysis method, Int. J. Appl. Math. Mech., 8 (2012) 51-75.

[18] M. A. El-Tawil and S. N. Huseen, On the convergence of the q-homotopy analysis method, Int. J. Contemp. Math. Sci., 8 (2013) 481-497.

[19] S. Momani and Z. M. Odibat, Analytical solution of a timefractional Navier-Stokes equation by Adomain decomposition method, Appl. Math. Comp., 177 (2006) 488-494.

[20] N. A. Khan, A. Ara, S. A. Ali and A. Mahmood, Analytic study of Navier-Stokes equation with fractional order using He's homotopy perturbation and variational iteration method, International Journal of Nonlinear Science and Numerical Simulation, 10(9) (2009) 1127-1134.

[21] A. A. Ragab, K. M. Hemida, M. S. Mohamed and M. A. Abd El Salam, Solution of time- fractional Navier-Stokes Equation by using Homotopy Analysis method, Gen. Math. Notes, 13(2) (2012) 13-21.

[22] S. Kumar, D. Kumar, S. Abbasbandy and M. M. Rashidi, Analytical solution of fractional Navier-Stokes equation by using modified Laplace decomposition method, Ain Shams Engineering Journal, 5 (2014) 569-574.

[23] D. Kumar, J. Singh and S. Kumar, A fractional model of NavierStokes equation arising in unsteady flow of a viscous fluid, Journal of the Association of Arab Universities for Basic and Applied Science, 17 (2015) 14-19.

[24] K. Wang and S. Liu, Analytical study of time-fractional NavierStokes equation by using transform methods, Advances in Difference Equations, 61 (2016) 1-12, DOI 10.1186/s13662-0160783-9.

[25] Y. Zhoua and Li Peng, On the time-fractional Navier-Stokes equations, Comp. Math. Appl., 73 (2017) 874-891.

[26] I. Podlubny, Fractional Differential Equations, Academic Press, San Diego, 1999. 